\title{
Numerical investigation of the effects of shock tube geometry on the propagation of an ideal blast wave profile
}

\author{
X. D. $\mathrm{Li}^{1}$ (1) - Z. M. Hu${ }^{1,2}$ - Z. L. Jiang ${ }^{1}$
}

Received: 4 September 2015 / Revised: 6 February 2016 / Accepted: 17 February 2017 / Published online: 1 March 2017

(C) Springer-Verlag Berlin Heidelberg 2017

\begin{abstract}
Bio-shock tubes (BSTs) can approximately simulate the typical blast waves produced by nuclear or chemical charge explosions for use in biological damage studies. The profile of an ideal blast wave in air is characterized by the overpressure, the negative pressure, and the positive pressure duration, which are determined by the geometric configurations of BSTs. Numerical experiments are carried out using the Eulerian equations by the dispersion-controlled dissipative scheme to investigate the effect of different structural components on ideal blast waveforms. The results show that cylindrical and conical frustum driver sections with an appropriate length can produce typical blast wave profiles, but a flattened peak pressure may appear when using a tube of a longer length. Neither a double-expansion tube nor a shrinkage tube set in BSTs is practical for the production of an ideal blast waveform. In addition, negative pressure recovery will occur, exceeding the ambient pressure with an increase in pressure in the vacuum section.
\end{abstract}

Keywords Blast wave profile - Biological shock tube . Blast overpressure $\cdot$ Geometrical configuration

Communicated by C. Needham and A. Higgins.

Z. M. Hu

huzm@imech.ac.cn

1 State Key Laboratory of High Temperature Gas Dynamics, Institute of Mechanics, Chinese Academy of Sciences, Beijing 100190, China

2 School of Engineering Science, University of Chinese Academy of Sciences, Beijing 100049, China

\section{Introduction}

Blast overpressure (BOP) is defined as the increased pressure over ambient atmospheric pressure that occurs in civilian and military detonations or from the firing of weapons. Exposure to incident BOP waves is known to damage the hollow gas-filled organs such as ears, lungs, and intestines [1-4]. Traumatic brain injury and blast-induced neurotrauma upon exposure to blast waves have also been confirmed in animal studies [5-9]. In order to reproduce the ideal blast wave profiles, the proper designs of these studies are appropriate to replicate field conditions in the laboratory.

The ideal overpressure wave resulting from an air blast in an open environment is described as a Friedlander waveform $[2,3,10]$. This waveform (Fig. 1) is characterized by a steep shock front, an initial positive phase, and a subsequent negative phase due to nonlinear decay, wherein the pressure falls below the ambient pressure. The typical blast wave profile is quantified by several factors such as overpressure, subambient pressure, positive pressure duration, and impulse.

To conduct explosion-simulation experiments in the laboratory, shock tubes have been extensively applied to the study of auditory and non-auditory damage for both physiological and pathological manifestation [2]. In such tubes, shock waves required for the measurements are driven either by high-pressure gases or by detonation of a fuel-oxidizer mixture. In particular, those tubes used to reproduce BOP waves for the study of biological damage are usually designated as biological shock tubes (BSTs) [12], which include compression-driven and blast-driven designs. Compressiondriven shock tubes $[2,8,10-13,16]$ avoid the safety concerns related to the storage and handling of high explosives and reduce the cost of test facilities to maintain, but they often fail to accurately represent the Friedlander waveform of freefield blast waves [5,6]. Blast-driven shock tubes $[14,15,17]$ 


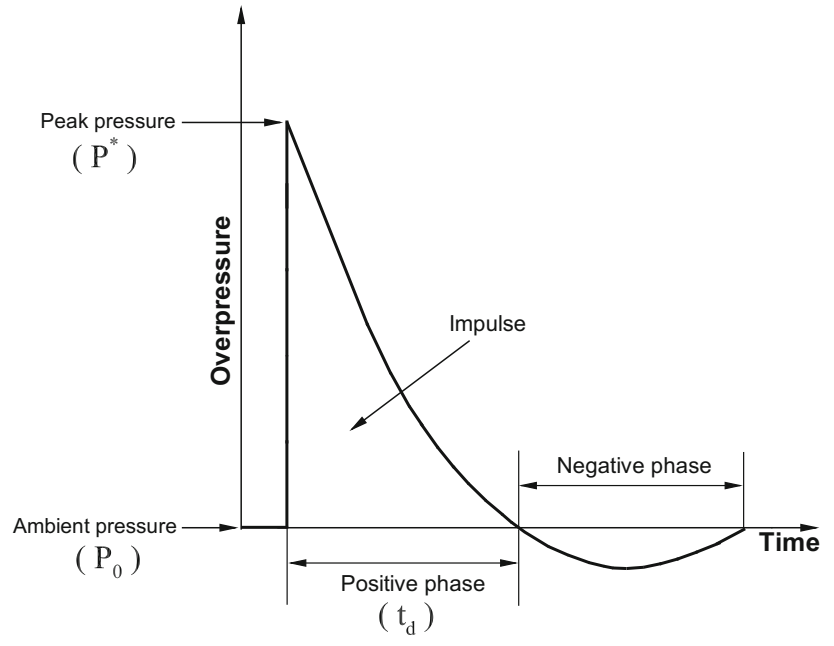

Fig. 1 Schematic diagram of an ideal blast wave profile with a positive phase (above ambient pressure) and a negative phase (below ambient pressure). $P^{*}$ is the peak pressure, $P_{0}$ is the ambient pressure, and $t_{\mathrm{d}}$ is the positive phase duration

can produce more realistic pressure-time profiles than the compression-driven ones, but their operation requires reliable facilities and is more dangerous due to the use of high explosive materials. Most current shock tube designs do not meet all requirements for fidelity of the pressure wave and accessibility in terms of facilities and cost [9]. The blast wave profile not only determines the survival of animal models, but also the acute biomechanical injuries and the chronic biochemical sequelae [16]. Therefore, it is of great importance to carefully design and operate bio-shock tubes to generate the ideal blast wave. However, little research has been focused on further understanding of the relationship between the ideal blast wave profile and the detailed geometric configuration of a shock tube.

In this paper, we have examined the effect of the geometric configuration of a BST on the propagation of an ideal blast wave profile. Several structures including a cylindrical driver section, a driving section with a front-conical tube, a double-expansion pipe, and a shrinkage tube are systematically investigated to determine the suitability of such structures for the production of typical blast waveforms. Furthermore, the influence of pressure variation in the vacuum section is also discussed.

\section{Models and numerical methodology}

\subsection{Geometrical models}

For this study, the BST is considered as a compressiondriven shock tube, in which the blast wave is produced by compressed air instead of an explosive mixture. The primary configuration of a biological shock tube, which is closely associated with the ideal blast waveform, consists

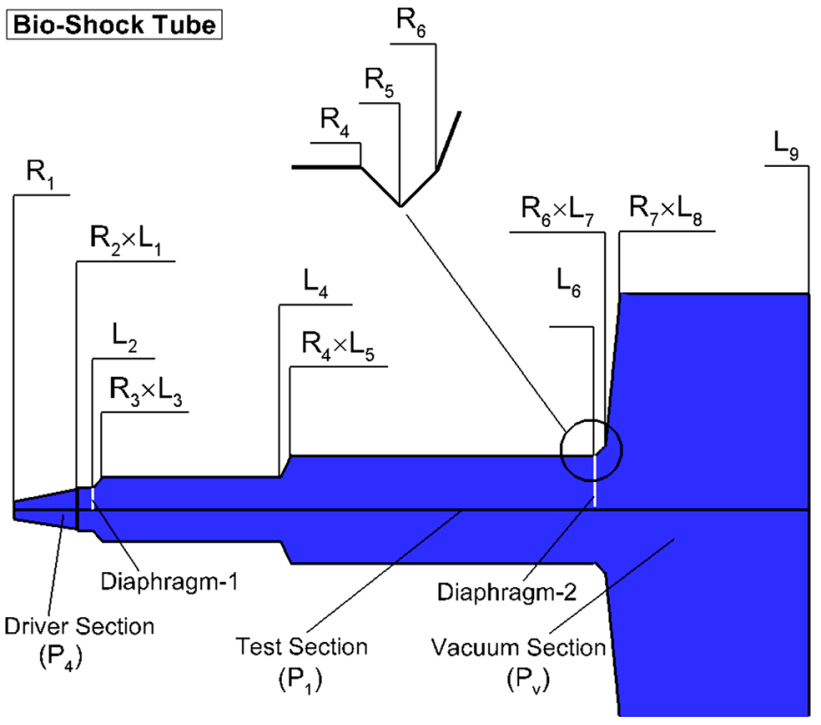

Fig. 2 The geometrical configuration of the bio-shock tube, mainly including a conical frustum driver section, an expansion section, a test section, a vacuum section, and a constricted tube marked by a circle. Two diaphragms are located at the end of the driver and vacuum sections. $R(\mathrm{~m}), L(\mathrm{~m})$ and $P(\mathrm{~atm})$ are, respectively, designated as the radius, length, and initial pressure of the bio-shock tube

of a driver section, a test section, an expansion section, and a vacuum section, as sketched in Fig. 2. The detailed parameters of $R, L$, and $P$ are, respectively, defined as the radius, the length, and the initial pressure in different components of BSTs. Several geometrical structures of BSTs considered will be investigated for the propagation of ideal blast wave profiles, such as a cylindrical driver section designated as the two radii $R_{1}=R_{2}$, a conical frustum driver section designated as the radii $R_{1}<R_{2}$, a double-expansion tube determined by the parameters of $R_{3}, R_{4}, L_{4}, L_{5}$, and a constricted tube positioned before the vacuum section determined by the parameters of $R_{4}, R_{5}, R_{6}$, which is shown in the enlarged drawing. The total length of the BST considered in this paper is $L_{9}=72 \mathrm{~m}$, and the test point is located at $40 \mathrm{~m}$.

Two diaphragms are separately located at the end of the driver section and the end of the test section, as shown in Fig. 2. When the first diaphragm is ruptured, the static highpressure gases in the driver section generates a strong main shock wave to the right and a rarefaction wave to the left. Once the shock wave arrives at the test section, the pressure at the test point increases sharply to reach the peak positive pressure and then decreases rapidly below the atmospheric pressure due to a reflected rarefaction wave catching up with the shock wave from the back end of the driver section. As a result, the typical blast wave profile, including positive pressure, negative pressure, and positive pressure duration, is formed. The shock wave continues to propagate away from the driver section, breaks the second diaphragm, and enters the vacuum section, where the high pressure is reduced. The 
reason for setting the second diaphragm before the vacuum section is that the initial static pressures at the test section $\left(P_{1}\right)$ and the vacuum section $\left(P_{\mathrm{v}}\right)$ for numerical simulation are not equal to each other. If the initial pressures $P_{1}=P_{\mathrm{v}}$, the second diaphragm is automatically not available for the shock tubes.

In Table 1, all the fixed parameters are listed for reference, including the different lengths of structural components ( $L_{4}, L_{5}, L_{6}, L_{7}, L_{8}$, and $L_{9}$ ), the corresponding radii $\left(R_{2}, R_{4}, R_{6}\right.$, and $\left.R_{7}\right)$, the initial low pressure $P_{1}$ in the test section, and the initial high pressure $P_{4}$ in the driver section. The fixed total length $\left(L_{9}\right)$ and test section radius $\left(R_{4}\right)$ of the shock tube may leave limited flexibility for alternate designs, but the present study of BSTs is focused on determining if the production of ideal blast waveforms is feasible with the possible geometrical configurations or not, and the following results will be applicable for other total lengths of shock tubes, because the sizes of the structural components of the shock tube are the major factors. The initial static pressure $P_{1}=0.5 \mathrm{~atm}$ in the test section, corresponding to the height of approximately $5000 \mathrm{~m}$, can be regarded as a blast environment at high altitude or on continental plateaus rather than at sea level. Although such a low pressure $\left(P_{1}\right)$ in the atmospheric environment-possibly causing stress on an animal subject independent of the blast loading - is an issue and may prevent the exact design proposed in this paper from being used for live biological specimens, high or low initial pressures set in the test section for numerical experiments mainly have an influence on the positive phase duration instead of the typical blast wave profile. In addition, a fixed driver pressure $\left(P_{4}\right)$ is used to reduce excessive calculation variables, which was not the main issue of concern in the present study.
In Table 2, the other unfixed parameters for the calculations, such as $L_{1}, L_{2}, L_{3}, R_{1}, R_{3}, R_{5}$, and $P_{\mathrm{v}}$, will be used to observe the blast waveform in different cases, which involve the configuration of a driver section, a double-expansion tube, a shrinkage tube, and the pressure variation in the vacuum section.

\subsection{Governing equations}

All of the calculations are performed using several simplifying assumptions, including an ideal gas, inviscid compressible flow, and two-dimensional axisymmetric flow. The inviscid simplification is justified because the gas viscosity has little effect on the structure of the shock wave. Another reason is that the thickness of the viscous wall boundary layer in the test section of bio-shock tubes is much smaller than the mainstream area. To reduce the computational cost, the flows in BSTs are numerically simulated using 2-D axisymmetric Euler equations. The governing equations can be written in a conservation form, which are expressed as

$$
\frac{\partial \mathbf{U}}{\partial t}+\frac{\partial \mathbf{F}}{\partial x}+\frac{\partial \mathbf{G}}{\partial y}+\frac{\mathbf{S}}{y}=0 .
$$

Here, $\mathbf{U}$ is the unknown state vector, $\mathbf{F}$ and $\mathbf{G}$ are the inviscid flux vectors along the $x$ and $y$ directions, respectively, and $\mathbf{S}$ is the source vector, where

$$
\begin{aligned}
\mathbf{U} & =[\rho, \rho u, \rho v, \rho E]^{\mathrm{T}}, \\
\mathbf{F} & =\left[\rho u, \rho u^{2}+p, \rho u v,(\rho E+p) u\right]^{\mathrm{T}}, \\
\mathbf{G} & =\left[\rho v, \rho u v, \rho v^{2}+p,(\rho E+p) v\right]^{\mathrm{T}}, \\
\mathbf{S} & =\left[\rho v, \rho u v, \rho v^{2},(\rho E+p) v\right]^{\mathrm{T}} .
\end{aligned}
$$

\begin{tabular}{|c|c|c|c|c|c|c|c|c|c|c|c|}
\hline$L_{4}(\mathrm{~m})$ & $L_{5}(\mathrm{~m})$ & $L_{6}(\mathrm{~m})$ & $L_{7}(\mathrm{~m})$ & $L_{8}(\mathrm{~m})$ & $L_{9}(\mathrm{~m})$ & $R_{2}(\mathrm{~m})$ & $R_{4}(\mathrm{~m})$ & $R_{6}(\mathrm{~m})$ & $R_{7}(\mathrm{~m})$ & $P_{1}(\mathrm{~atm})$ & $P_{4}(\mathrm{~atm})$ \\
\hline 20 & 21 & 51 & 52 & 53.5 & 72 & 0.2 & 0.5 & 0.6 & 2.0 & 0.5 & 50 \\
\hline
\end{tabular}

Table 1 Geometric sizes and initial pressure for reference

Table 2 Different geometric and pressure parameters given for each calculation

\begin{tabular}{clllllll}
\hline Cases & $L_{1}(\mathrm{~m})$ & $L_{2}(\mathrm{~m})$ & $L_{3}(\mathrm{~m})$ & $R_{1}(\mathrm{~m})$ & $R_{3}(\mathrm{~m})$ & $R_{5}(\mathrm{~m})$ & $P_{\mathrm{v}}(\mathrm{atm})$ \\
\hline 1 & 0.5 & 1.0 & 2.0 & 0.2 & 0.5 & 0.5 & 0.25 \\
2 & 1.0 & 1.5 & 2.5 & 0.2 & 0.5 & 0.5 & 0.25 \\
3 & 1.0 & 4.0 & 5.0 & 0.2 & 0.5 & 0.5 & 0.25 \\
4 & 0.8 & 1.5 & 2.5 & 0.05 & 0.5 & 0.5 & 0.25 \\
5 & 1.0 & 1.5 & 2.5 & 0.05 & 0.5 & 0.5 & 0.25 \\
6 & 1.2 & 1.5 & 2.5 & 0.05 & 0.5 & 0.5 & 0.25 \\
7 & 1.0 & 1.5 & 2.5 & 0.2 & 0.3 & 0.5 & 0.25 \\
8 & 1.0 & 1.5 & 2.5 & 0.2 & 0.5 & 0.25 & 0.25 \\
9 & 1.0 & 1.5 & 2.5 & 0.2 & 0.5 & 0.5 & 0.10 \\
10 & 1.0 & 1.5 & 2.5 & 0.2 & 0.5 & 0.5 & 0.35 \\
11 & 1.0 & 1.5 & 2.5 & 0.2 & 0.5 & 0.5 & 0.50 \\
\hline
\end{tabular}


The scalar $E$ is the total energy per unit mass, which has a relationship with the pressure to close the above equations, i.e.,

$p=(\gamma-1)\left(\rho E-\frac{1}{2} \rho\left(u^{2}+v^{2}\right)\right)$,

where the ratio of specific heat $\gamma$ is 1.4 .

\subsection{Numerical methods}

The spatial discretization scheme adopts the DCD scheme [18] in this study, which has been confirmed to be a satisfactory numerical technique for shock-capturing and complex flow simulation $[19,20]$. The inviscid fluxes are solved by the Steger-Warming splitting method, and the temporal discretization is obtained by the second-order Runge-Kutta explicit method. The boundary condition is a non-viscous slip wall, i.e.,

$\frac{\partial u}{\partial x}=0, \frac{\partial v}{\partial y}=0$

The velocity at the initial instant is zero and the initial pressures are given in Table 1, which are imposed to the initialization for all grids. The initial pressure $P_{\mathrm{v}}$ in the vacuum section is given in Table 2 for the eleven test cases.

\subsection{Grid-independence verification}

Four groups of computational grids are used for the gridindependence verification, and the test point is located at 40.0 $\mathrm{m}$ along the central axis of BSTs. The test numerical results are calculated using the same geometric sizes and the same initial pressure, which is referred to as case 2 in Table 2. In Fig. 3, four blast wave profiles exhibit a similar maximum of pressures for both coarse and fine grids, while the two waveforms, respectively, associated with $3500 \times 50$ and $3500 \times 100$ grids are almost the same and the peak pressure only changes $0.1 \%$ for the finest grid $3500 \times 100$, as given in Table 3. In other words, the finest grids are sufficient to resolve the equations for capturing the blast waveforms in the subsequent computations.

\section{Results and discussion}

\subsection{Driver section with a cylindrical tube}

Three different lengths of the cylindrical driver sections are studied for the blast wave profiles, the parameters of which, respectively, correspond to case 1 , case 2 , and case 3 in Table 2. Figure 4 shows the typical pressure-time profiles for the blast waves and the geometric configuration

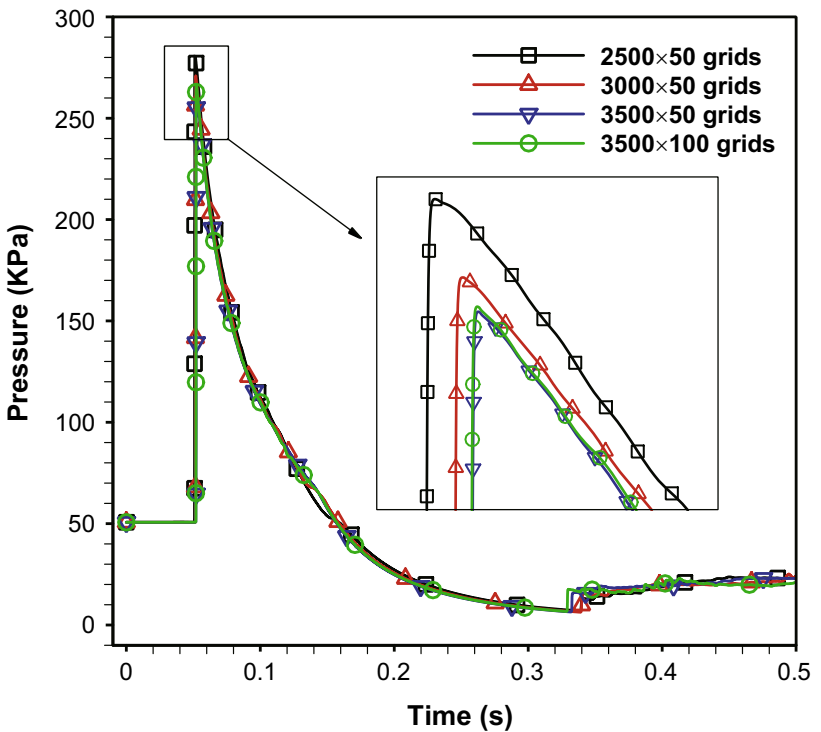

Fig. 3 The blast waveforms at the test point for the coarse and fine grids

Table 3 The peak pressures for the coarse and fine grids

\begin{tabular}{lll}
\hline Groups & Grids & Peak pressure $(\mathrm{kPa})$ \\
\hline 1 & $2500 \times 50$ & 280.54 \\
2 & $3000 \times 50$ & 269.86 \\
3 & $3500 \times 50$ & 266.69 \\
4 & $3500 \times 100$ & 266.33 \\
\hline
\end{tabular}

located at the top right corner of each plot. The cylindrical driver sections with the small scale $L_{2}=1.0 \mathrm{~m}$ and the medium scale $L_{2}=1.5 \mathrm{~m}$ both exhibit a blast waveform of the desired profile, as shown in Fig. 4a, b, respectively. However, no sharp wave crest can be seen in the driver section with $L_{2}=4.0 \mathrm{~m}$, which is shown in Fig. 4c. The peak pressures obtained from the small- to large-scale driver sections are 231.3, 266.7, and $269.8 \mathrm{kPa}$, respectively, and accordingly the positive pressure durations are 0.092, 0.104, and 0.090 seconds. Such high peak pressures and long pulse durations in these numerical simulations are mainly determined by the initial pressure in the driver section $\left(P_{4}=50 \mathrm{~atm}\right)$ and the pressure in the test section $\left(P_{1}=0.5 \mathrm{~atm}\right)$, which may prevent an exact design and fabrication proposed in the paper from being applied for live biological specimens. However, the same shock tube designs can be modified to obtain the blast loads of smaller charges and lower positive phase durations, provided the driver pressure $P_{4}$ is given by a lower initial static pressure and the cylindrical driver section is designed to a shorter size.

Figure 5 shows the $x-t$ wave system diagrams for a time evolution of the blast wave in $0.4 \mathrm{~s}$, which are obtained by the 

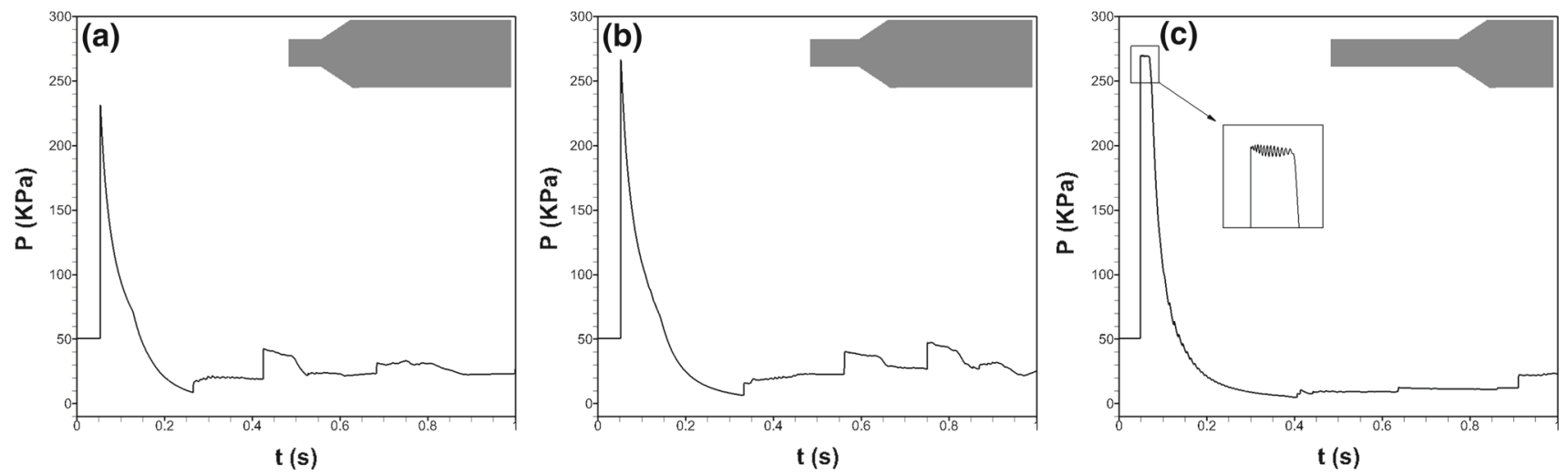

Fig. $4 p-t$ profiles for the cylindrical driver sections. a The small scale $L_{2}=1.0 \mathrm{~m}$. b The medium scale $L_{2}=1.5 \mathrm{~m}$. c The large scale $L_{2}=4.0 \mathrm{~m}$
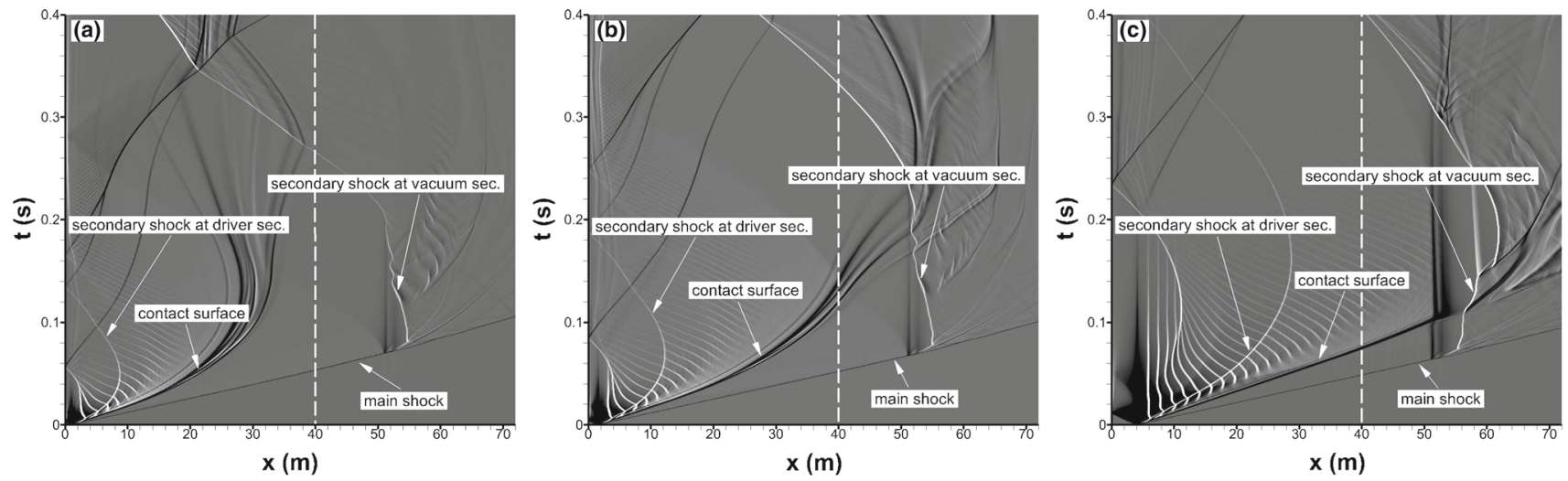

Fig. $5 x-t$ wave system diagrams associated with the schlieren visualization for the cylindrical driver sections. a The small scale $L_{2}=1.0 \mathrm{~m}$. b The medium scale $L_{2}=1.5 \mathrm{~m}$. c The large scale $L_{2}=4.0 \mathrm{~m}$

flowfield schlieren visualization. It can be observed that the wave systems in the BSTs include a main shock wave generated at the first diaphragm position, a secondary shock wave at the driver section, a contact surface between the main and secondary shock waves, another secondary shock produced at the second diaphragm, and other complex reflected waves. The three $p-t$ profiles in Fig. $4 \mathrm{a}-\mathrm{c}$, respectively, correspond to the position of $40.0 \mathrm{~m}$ in the test section, as shown along the dashed line in Fig. 5a-c. In the $x-t$ diagrams, it can be seen clearly that the main shock arrived at the test point leading to a peak pressure of the $p-t$ profile, and then the high positive pressure decreases below the initial ambient pressure because the reflected rarefaction wave comes from the closed end of the driver section. As time progresses, the negative pressure begins to rise again, due to the recompression of an upstream secondary shock produced at the second diaphragm set before the vacuum section. When the highpressure gas flows through the second diaphragm, it causes a new shock wave at the diaphragm to match the pressure difference in the flowfield because of unequal static pressures on both sides of the second diaphragm. Comparing Figs. $4 \mathrm{c}$ and $5 \mathrm{c}$ indicates that a flattening of the peak pressure profile might be observed if the total driver-section length is long enough, due to the fact that the rarefaction wave has not yet caught up to the main shock wave.

\subsection{Driver section with a conical frustum tube}

Three driver sections with different sizes of conical frustum tubes are investigated for the production of ideal blast wave profiles, which corresponds to case 4 , case 5 , and case 6 in Table 2, respectively. The three configurations have the same total length of driver section with $L_{2}=1.5 \mathrm{~m}$ and the same radius with $R_{1}=0.05 \mathrm{~m}$, whereas the conical sections are of different lengths $L_{1}=0.8,1.0$, and $1.2 \mathrm{~m}$, as shown at the top right corner of Fig. $6 \mathrm{a}-\mathrm{c}$. However, there are no obvious distinctions for the blast waveforms among the satisfactory $p-t$ profiles when using a conical frustum tube. The numerical peak pressures obtained from the small- to large-scale driver sections are $257.4,254.5$, and $251.9 \mathrm{kPa}$, respectively, and the positive time durations for the three cases are all close to $0.1 \mathrm{~s}$ in the simulations.

In Fig. $7 \mathrm{a}-\mathrm{c}$, the $x-t$ wave system diagrams associated with the schlieren visualizations are quite similar, showing 

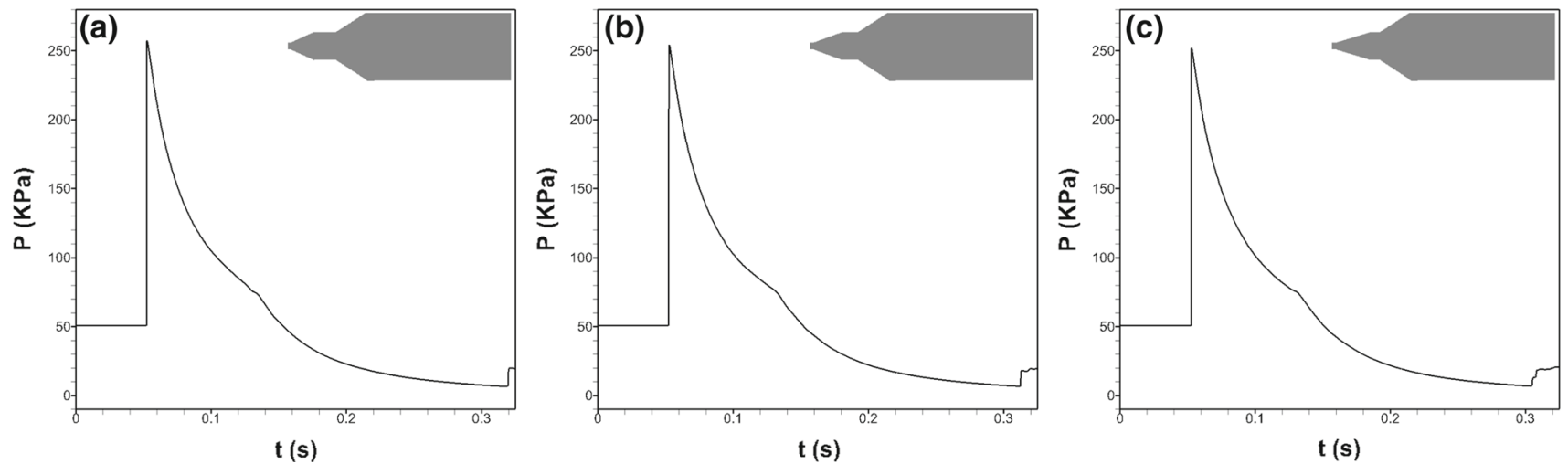

Fig. $6 p-t$ profiles for the conical frustum driver sections. a The small scale $L_{1}=0.8 \mathrm{~m}$. b The medium scale $L_{1}=1.0 \mathrm{~m}$. $\mathbf{c}$ The large scale $L_{1}=1.2 \mathrm{~m}$
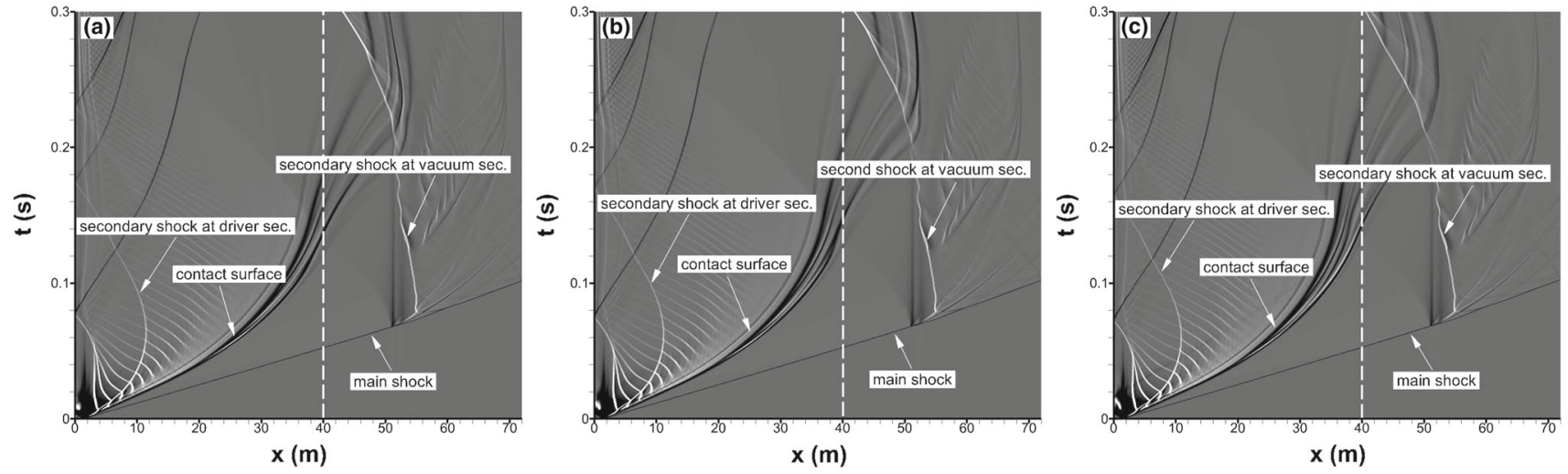

Fig. $7 x-t$ wave system diagrams associated with the schlieren visualization for the conical frustum driver sections. a The small scale $L_{1}=0.8 \mathrm{~m}$. b The medium scale $L_{1}=1.0 \mathrm{~m}$. c The large scale $L_{1}=1.2 \mathrm{~m}$

a main shock wave, two secondary shock waves generated, respectively, from the driven section and the second diaphragm, a contact surface and other complex reflected waves. Compared with the $p-t$ profile for the cylindrical driver section in Fig. 4b, namely case 2 in Table 2, the driver section with a conical frustum structure can also produce an ideal blast wave profile, but it has little effect on the production of the typical blast waveform. It indicates that the total length $\left(L_{2}\right)$ of the driver section has a significant impact on the ideal blast wave profile rather than a conical frustum configuration in the driver section.

\subsection{Double-expansion tube}

The double-expansion tube is set between the driver and test sections, which is described by the geometrical parameters, i.e., $R_{3}, R_{4}, L_{4}$, and $L_{5}$, as shown briefly in Fig. 2. For case 7 in Table 2, we have tentatively reduced the geometrical radius $R_{3}$ to be $0.3 \mathrm{~m}$ and located the double-expansion tube at $L_{4}=20 \mathrm{~m}$ in the BST for numerical simulation. It can be obviously seen that two peak pressures appear in the unsatisfactory $p-t$ profile, as shown in the enlarged plot in Fig. 8a with the geometry used in the calculation shown at the top right corner. The main reason for such a phenomenon in this case is that there is a weak compression wave generated at the double-expansion position following behind the main shock wave, leading to a recompression process at the test point, which can be carefully observed from the $x-t$ wave system profile marked with a circle in Fig. 8b. Therefore, it shows that the double-expansion tube might produce unnecessary compression or shock waves to disrupt an ideal blast waveform when set in bio-shock tubes.

\subsection{Shrinkage tube before the vacuum section}

To examine the effect of a shrinkable device on pressurerelief protection when the strong main shocks propagate into the vacuum section, a shrinkage tube determined by the geometrical parameters of $R_{5}=0.25 \mathrm{~m}, L_{6}=50 \mathrm{~m}$, and $L_{7}=51 \mathrm{~m}$ is positioned before the vacuum section, as simply described and marked by a circle in Fig. 2. For the production of an ideal blast waveform, the $p-t$ profile will be worse with a given shrinkage tube than without, which is 


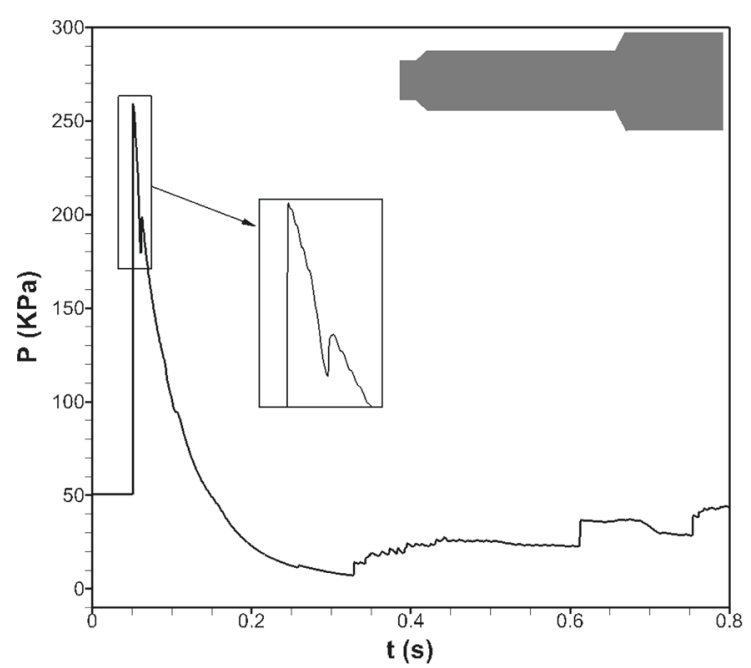

(a)

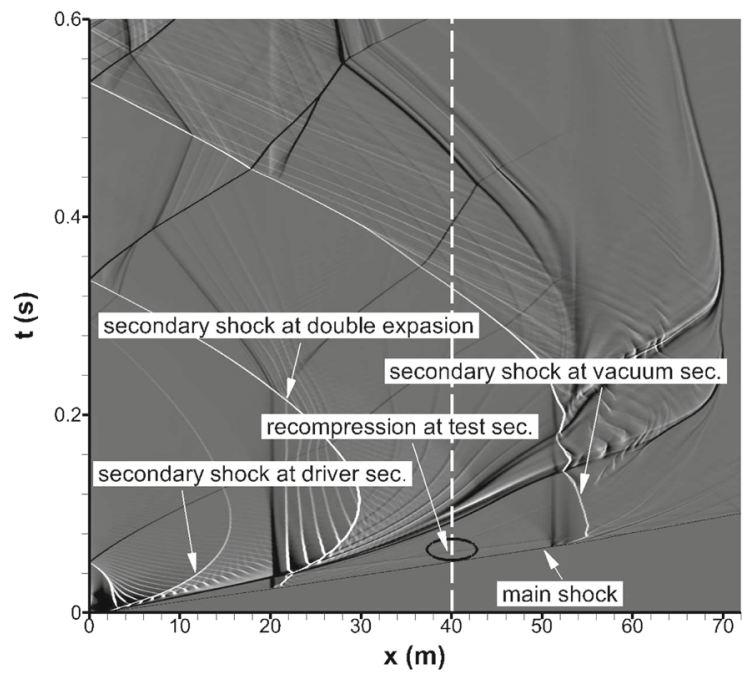

(b)

Fig. $8 p-t$ profile and the wave system diagram associated with the schlieren visualization for the double-expansion tube. a $p-t$ profile. b $x-t$ profile

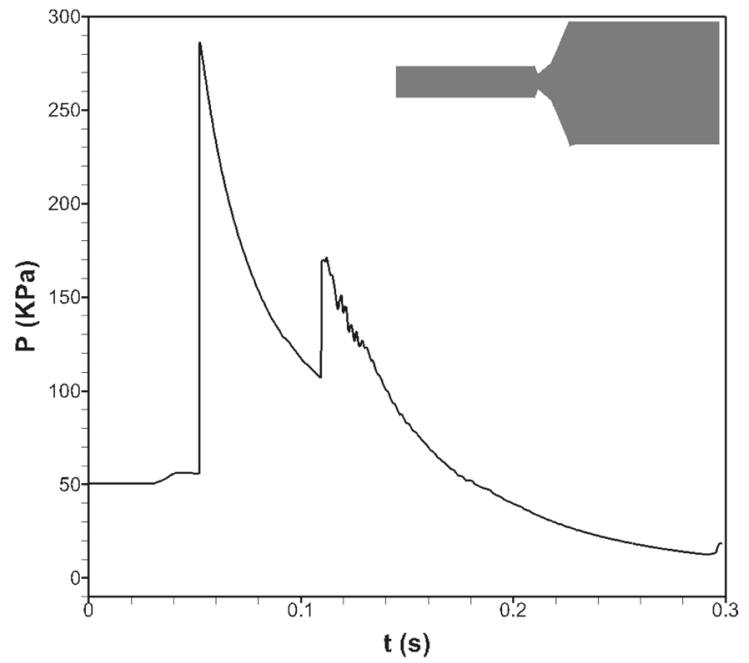

(a)

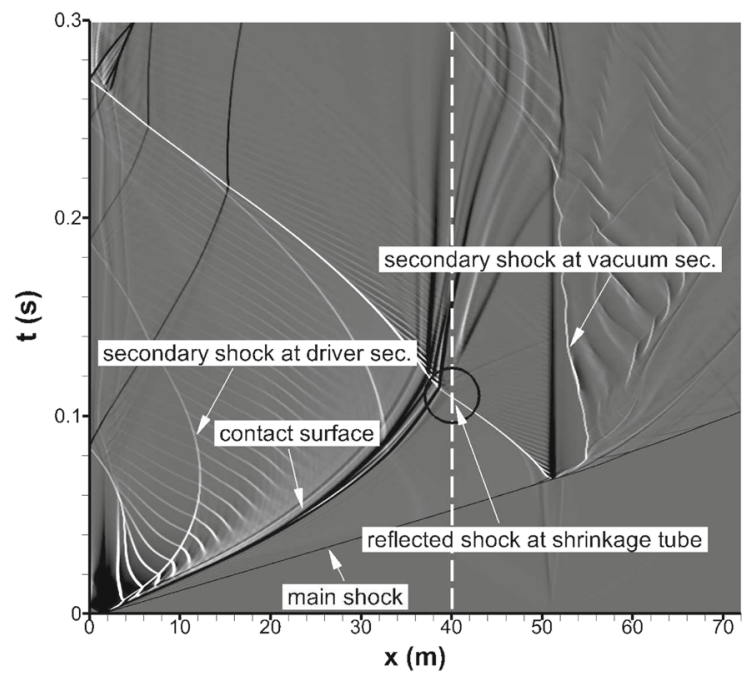

(b)

Fig. $9 p-t$ profile and the wave system diagram associated with the schlieren visualization for the shrinkage tube. a $p-t$ profile. $\mathbf{b} x-t$ profile

clearly shown in Fig. 9a and the corresponding calculation parameters can be obtained from case 8 in Table 2. Compared to the benchmark test without a conical frustum, a doubleexpansion or a shrinkage tube in case 2 , the only difference in case 8 is that the radius $R_{5}$ located before the vacuum section is reduced by half. Unfortunately, the non-ideal $p-t$ profile in Fig. 9a exhibits two peak pressures for the unsatisfactory blast wave, because a reflected shock wave comes from the constricted tube for recompression at the test position, which can be observed clearly from a marked circle in Fig. 9b. Consequently, it seems reasonable to avoid setting the shrinkage structure before the vacuum section of a BST, since the unsat- isfactory blast waveforms can be attributed almost entirely to the effects of an unnecessary reflected shock.

\subsection{Pressure change in the vacuum section}

In order to investigate the effect of pressure change in the vacuum section for the production of an ideal blast wave profile, four $p-t$ profiles in Fig. 10 are calculated using the same cylindrical driver section with a medium scale $L_{2}=1.5 \mathrm{~m}$ for the BSTs, the geometrical parameters of which, respectively, correspond to case 2 , case 9 , case 10 , and case 11 in Table 2. The ideal blast waveform as a benchmark result with $P=0.25 \mathrm{~atm}$ for case 2 is presented by the solid line with 


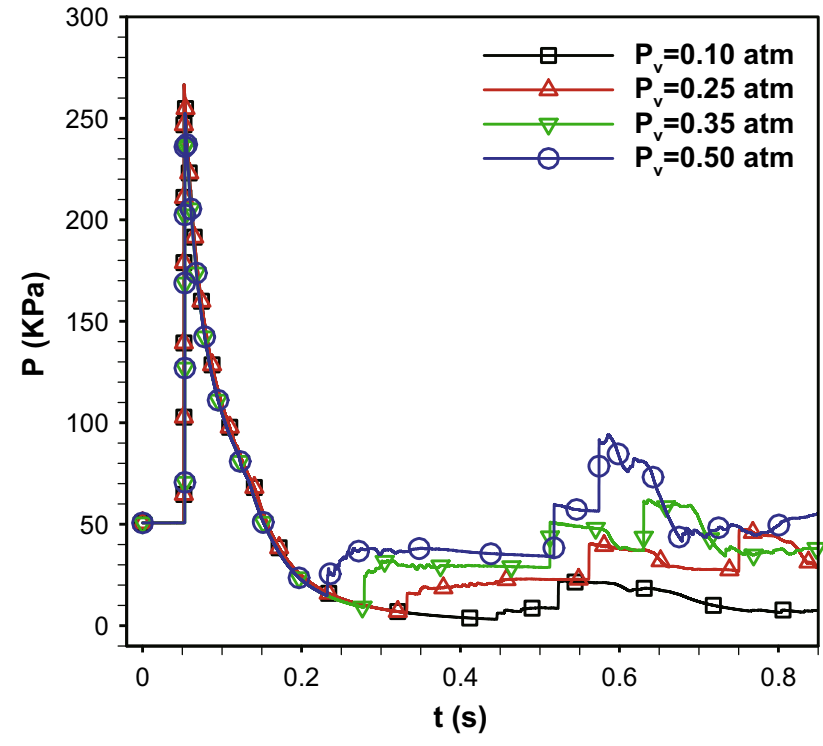

Fig. $10 p-t$ profiles for different pressures in the vacuum section

triangles in Fig. 10, while the other initial low pressures in the vacuum section are $0.10 \mathrm{~atm}$ for case $9,0.35 \mathrm{~atm}$ for case 10 , and $0.50 \mathrm{~atm}$ for case 11 , respectively. Comparison with the calculated results show that the four cases have the same ideal blast wave profiles and positive pressure durations, but differ in the negative pressure variations in the vacuum section. In other words, the higher the initial low pressure set in the vacuum section, the more quickly the recovery of the negative pressure; even the recovering low pressure may exceed an initial ambient pressure to be an overpressure, such as the numerical description of the solid line with circles for case 11.

\section{Conclusions}

A numerical investigation is performed to study the geometric configurations of a bio-shock tube capable of generating ideal blast wave profiles, and the findings are summarized as follows. First, the total length of driver section should not be too long because of a probable pressure plateau appearing in the blast wave profile. Second, the cylindrical and conical frustum driver sections can both produce a typical blast waveform when the driver sections are of an appropriate total length; the same design can be modified to obtain smaller blast loads and shorter positive phase durations from a lower initial driver pressure and a shorter scale of cylindrical driver section. Thirdly, double-expansion and shrinkage tubes set in the BST have a significant impact on the blast waveforms, which are not desirable for the production of an ideal blast wave profile. In addition, the negative pressure recovery occurs more quickly with the higher initial low pres- sure in the vacuum section and may increase to approach the ambient or positive pressures.

The above results for simulating the blast wave profiles are based on the inviscid axisymmetric flows and some simple configurations of a bio-shock tube. Despite this limitation, these findings may, to some extent, shed new light on identifying an appropriate geometrical structure for the BST, which can produce an ideal blast wave with a short duration and a smooth profile. Further investigations and experimental validations are required to ascertain the details of the geometric sizes of BSTs necessary to generate an ideal blast wave profile.

Acknowledgements This work is supported by the National Natural Science Foundation of China under Grant No. 11532014

\section{References}

1. Philips, Y.Y.: Primary blast injuries. Ann. Emerg. Med. 15(12), 1446-1450 (1986). doi:10.1016/S0196-0644(86)80940-4

2. Elsayed, N.M.: Toxicology of blast overpressure. Toxicology 121, 1-15 (1997). doi:10.1016/S0300-483X(97)03651-2

3. Mayorga, M.A.: The pathology of primary blast overpressure injury. Toxicology 121, 17-28 (1997). doi:10.1016/ S0300-483X(97)03652-4

4. Stuhmiller, J.H.: Biological response to blast overpressure: A summary of modeling. Toxicology 121, 91-103 (1997). doi:10.1016/ S0300-483X(97)03658-5

5. Chavko M., Koller W.A., Prusaczyk W.K., McCarron R.M.: Measurement of blast wave by a miniature fiber optic pressure transducer in the rat brain. J. Neurosci. Methods 159, 277-281 (2007). doi:10.1016/j.jneumeth.2006.07.018

6. Chavko, M., Watanabe, T., Adeeb, S., Lankasky, J., Ahlers, S.T., McCarron, R.M.: Relationship between orientation to a blast and pressure wave propagation inside the rat brain. J. Neurosci. Methods 195, 61-66 (2011). doi:10.1016/j.jneumeth.2010.11.019

7. Ling, G., Bandak, F., Armonda, R., Grant, G., Ecklund, J.: Explosive blast neurotrauma. J. Neurotrauma 26(6), 815-825 (2009). doi:10.1089/neu.2007.0484

8. Skotak, M., Wang, F., Alai, A., Holmberg, A., Harris, S., Switzer, R.C., Chandra, N.: Rat injury model under controlled field-relevant primary blast conditions: Acute response to a wide range of peak overpressure. J. Neurotrauma 30(13), 1147-1160 (2013). doi:10. 1089/neu.2012.2652

9. Nakagawa, A., Manley, G.T., Gean, A.D., Ohtani, K., Armonnda, R., Tsukamoto, A., Yamamoto, H., Takayama, K., Tominaga, T.: Mechanisms of primary blast-induced traumatic brain injury: Insights from shock-wave research. J. Neurotrauma 28(6), 11011119 (2011). doi:10.1089/neu.2010.1442

10. Chandra, N., Ganpule, S., Kleinschmit, N.N., Feng, R., Holmberg, A.D., Sundaramurthy, A., Selvan, V., Alai, A.: Evolution of blast wave profiles in simulated air blasts: experiment and computational modeling. Shock Wave 22, 403-415 (2012). doi:10.1007/ s00193-012-0399-2

11. Richmond, D.R., Clare, V.R., Goldizen, V.C., Pratt, D.E., Sanchez, R.T., White, C.S.: Biological effects of overpressure. II. A shock tube utilized to produce sharp-rising overpressure of 400 milliseconds duration and its employment in biomedical experiments. Aerospace Med. 32, 997-1008 (1961) 
12. Wang, Z.G., Sun, L.Y., Yang, Z.H., Leng, H.G., Jiang, J.X., Yu, H.R., Gu, J.H., Li, Z.F.: Development of serial bio-shock tubes and their application. Chin. Med. J. 111(2), 109-113 (1998)

13. Leng, H.G., Wang, Z.G., Yang, Z.H., Li, X.Y., Yu, H.R., Gu, J.H., Li, Z.F., Li, Z.H.: A biological shock tube and an experimental study on animal tolerance to blast wave. Explos. Shock Waves 13(3), 272-279 (1993). (in Chinese)

14. Courtney, M.W., Courtney, A.C.: A table-top blast driven shock tube. Rev. Sci. Instrum. 81(12), 126103 (2010). doi:10.1063/1. 3518970

15. Courtney, A.C., Andrusiv, L.P., Courtney, M.W.: Oxy-acetylene driven laboratory scale shock tubes for studying blast wave effects. Rev. Sci. Instrum. 83, 045111 (2012). doi:10.1063/1.3702803

16. Sundaramurthy, A., Chandra, N.: A parametric approach to shape field-relevant blast wave profiles in compressed-gas-driven shock tube. Front Neurol. 5, 253 (2014). doi:10.3389/fneur.2014.00253

17. Stewart, J.B., Pecora, C.: Explosively driven air blast in a conical shock tube. Rev. Sci. Instrum. 86(3), 035108 (2015). doi:10.1063/ 1.4914898
18. Jiang, Z.L.: On dispersion-controlled principles for non-oscillatory shock-capturing schemes. Acta Mech. Sin. 20(1), 1-15 (2004). doi:10.1007/BF02484239

19. Jiang, Z.L., Takayama, K.: An investigation into the validation of numerical solution of complex flowfields. J. Comput. Phys. 151, 479-497 (1999). doi:10.1006/jcph.1999.6186

20. Hu, Z.M., Wang, C., Jiang, Z.L., Khoo, B.C.: On the numerical technique for the simulation of hypervelocity test flows. Comput. Fluids 106, 12-18 (2015). doi:10.1016/j.compfluid.2014.09.039 